\title{
Indicadores socioeconômicos e de saúde da Atenção Básica nos municípios da região metropolitana de Belo Horizonte
}

\section{Socioeconomic and health indicators of primary health care in the metropolitan region of Belo Horizonte}

Ana Cristina Viana Campos ${ }^{1}$, Carolina Marques Borges ${ }^{1}$, Cláudio Rodrigues Leles ${ }^{2}$, Andréa Maria Duarte Vargas $^{3}$, Efigênia Ferreira e Ferreira ${ }^{3}$

\section{RESUMO}

Objetivo: investigar a associação entre os indicadores socioeconômicos e de saúde da Atenção Básica nos municípios da Região Metropolitana de Belo Horizonte, Minas Gerais. Materias e Métodos: estudo ecológico com utilização de dados secundários referentes à Região Metropolitana de Belo Horizonte. As variáveis dependentes foram os indicadores de saúde geral e de saúde bucal da Atenção Básica. As variáveis exploratórias foram taxa de alfabetização, Índice de Desenvolvimento Humano, Índice de Gini e Índice Mineiro de Responsabilidade Social. A análise de Cluster Hierárquico foi realizada para segmentação dos municípios a partir dos indicadores socioeconômicos, por meio do método Between-linkage group e a medida Distância Euclidiana Quadrática. A comparação dos indicadores de saúde da Atenção Básica entre os clusters foi feita através do teste $t$-Student, com nível de significância estabelecido em 5\%. Resultados: os municípios foram agrupados em dois clusters: 1) piores indicadores socioeconômicos ( $\mathrm{N}=23)$ e 2) melhores $(\mathrm{N}=10)$. O cluster 1 apresentou maiores cobertura da Estratégia de Saúde da Família e média anual de visitas domiciliares (76,6 \pm 32,$5 ; 6,1 \pm 24,6$, respectivamente) do que o cluster $2(70,4 \pm 27,9 ; 0,8 \pm 0,6$, respectivamente) $(\mathrm{p}<0,05)$. Em relação aos indicadores de Saúde Bucal não houve diferenças estatisticamente significantes entre os clusters. Conclusão: sugere-se que há uma associação entre municípios da região metropolitana de Belo Horizonte com piores indicadores socioeconômicos e maior cobertura da Saúde da Família e média de visita domiciliar. Descritores: Atenção primária à saúde. Indicadores básicos de saúde. Indicadores sociais.

\section{INTRODUÇÃO}

A Atenção Básica em saúde é caracterizada por um conjunto de ações individuais e coletivas que abrangem a promoção e a proteção da saúde, a prevenção de doenças e agravos, o diagnóstico, o tratamento, a reabilitação e a manutenção da saúde. É desenvolvida por meio do trabalho em equipe, dirigida às populações de territórios bem delimitados, pelos quais assume a responsabilidade sanitária ${ }^{1}$.

A partir da Norma Operacional Básica de 1996 (NOB-01/96), foi possível acompanhar as atividades desenvolvidas no âmbito da rede de serviços, identificando problemas prioritários que, se enfrentados adequadamente, propiciarão mudanças positivas na situação de saúde das populações ${ }^{2}$.

A Portaria $\mathrm{n}^{\mathrm{o}} 493$, de 13 de março de $2006^{3}$, estabeleceu os principais indicadores do Pacto de Atenção Básica, que passam a ser calculados a partir das bases nacionais dos diversos sistemas do Ministério da Saúde. Os indicadores gerais são: a proporção da população coberta pela Saúde da Família e média anual de consultas médicas por habitante nas especialidades básicas. Um indicador complementar é a média mensal de visitas domiciliares por família. Para a área de saúde bucal, os indicadores constituem instrumento nacional de monitoramento e avaliação das ações e serviços de saúde bucal referentes à atenção básica. Dois indicadores são os principais: cobertura de primeira consulta odontológica programática e cobertura da ação coletiva de escovação dental supervisionada. Os indicadores complementares são: a média de procedimentos odontológicos básicos individuais e a proporção de procedimentos odontológicos especializados em relação às ações odontológicas individuais.

Diante das transformações econômicas,

\footnotetext{
${ }^{1}$ Programa de Pós-Graduação em Odontologia, Faculdade de Odontologia, Universidade Federal de Minas Gerais (UFMG), Belo Horizonte, MG, Brasil

${ }^{2}$ Departamento de Prevenção e Reabilitação Oral, Faculdade de Odontologia, Universidade Federal de Goiás, Goiânia (UFG), GO, Brasil ${ }^{3}$ Departamento de Odontologia Social e Preventiva, Faculdade de Odontologia, Universidade Federal de Minas Gerais (UFMG), Belo Horizonte, MG, Brasil

Contato: campos.acv@gmail.com,carolmborges@yahoo.com.br, crleles@odonto.ufg.br, vargasnt@task.com.br, efigeniaf@gmail.com
} 
sociais e demográficas no Brasil, com significativas repercussões nas condições de vida e trabalho da população e consequentemente em sua situação de saúde ${ }^{4}$; torna-se indispensável o estudo desses indicadores com o objetivo de reduzir as iniquidades de saúde ${ }^{5,6}$ geral e bucal ${ }^{7}$.

A problematização dessas questões poderá elucidar as diferenças entre os municípios e fornecer melhor conhecimento sobre o processo de implantação de políticas de saúde e contribuir para o aperfeiçoamento de estratégias de monitoramento e avaliação de sistemas e serviços de saúde ${ }^{8}$. Neste contexto, o presente artigo teve como objetivo investigar a associação entre os indicadores socioeconômicos de saúde da Atenção Básica nos municípios da Região Metropolitana de Belo Horizonte, Minas Gerais.

\section{MATERIAIS E MÉTODOS}

Estudo ecológico com a utilização de dados secundários obtidos no mês de março do ano de 2010 na página eletrônica do Sistema de Informação da Atenção Básica 2006, oriundos de Sistemas de Informações em Saúde (SIS) do Departamento de Informática do Sistema Único de Saúde - DATASUS e do Atlas Eletrônico do Desenvolvimento Humano que objetiva democratizar o acesso e aumentar a capacidade de análise sobre informações socioeconômicas relevantes.

A Região Metropolitana de Belo Horizonte (RMBH) é a terceira maior do país sendo composta por 34 municípios. As fronteiras municipais entre a capital Belo Horizonte e os outros municípios são virtuais, de tal modo que há um grande esforço dos governos municipais em buscar maior integração no planejamento das ações em todos os setores ${ }^{9}$.

A amostra de municípios foi escolhida por conveniência, seguindo outros estudos que enfatizam a importância de investigar a $\mathrm{RMBH}^{10-15}$.

As variáveis exploratórias foram quatro indicadores socioeconômicos: taxa de alfabetização (2000), Índice de Desenvolvimento Humano Municipal (IDH-M, 2000), Índice de Gini (2000), e Índice Mineiro de Responsabilidade Social (IMRS, 2005).

A taxa de alfabetização é o percentual de pessoas de 15 anos ou mais de idade que sabem ler e escrever pelo menos um bilhete simples, dividido pela população total da mesma faixa etária, em determinado espaço geográfico, no ano considerado. Essa variável expressa a situação educacional mínima da população.

O IDH-M é um indicador de desenvolvimento que agrega dados econômicos do Produto Interno Bruto (PIB) per capita real a outros dados relacionados com a educação (taxa de alfabetização de adultos e taxa de escolaridade combinada dos ensinos primário, secundário e superior) e à saúde (esperança média de vida à nascença). O Índice de Gini mede o grau de desigualdade existente na distribuição de indivíduos segundo a renda domiciliar per capita. Seu valor varia de 0 , quando supostamente não há desigualdade e a renda de todos os indivíduos tem o mesmo valor, a 1, quando a desigualdade é máxima e apenas um indivíduo detém toda a renda da sociedade e a renda de todos os outros indivíduos é nula.

O IMRS, elaborado pela Fundação João Pinheiro, é um indicador que expressa o nível de desenvolvimento de cada município mineiro. Foi desenvolvido para avaliar a situação desses municípios, contemplando nove dimensões, a saber: renda, saúde, educação, segurança pública, gestão, habitação e meio ambiente, cultura e desporto e lazer. Na construção do IMRS, os indicadores de cada tema são transformados em índices, com valores entre $0 \mathrm{e}$ 1, obtidos através da média ponderada dos índices dos indicadores que o compõem.

As variáveis independentes utilizadas para avaliar a Atenção Básica dos municípios mineiros foram os indicadores gerais e de saúde bucal da Atenção Básica disponíveis no DATASUS. Os três indicadores gerais foram: (i) cobertura da Estratégia Saúde da Família, (ii) média anual de consultas médicas por habitante nas especialidades e (iii) média mensal de visitas domiciliares por família. Quatro foram os indicadores de Saúde Bucal: (i) cobertura de primeira consulta odontológica programática, (ii) cobertura da ação coletiva de escovação dental supervisionada, (iii) média de procedimentos odontológicos básicos individuais e (iv) proporção de procedimentos odontológicos especializados em relação às ações odontológicas individuais).

A cobertura da Estratégia Saúde da Família (ESF) se refere à proporção da população coberta por esta política calculada por meio da divisão entre a população cadastrada no Sistema de Informação da Atenção Básica (SIAB) pelo total da população no mesmo local e período e, posteriormente multiplicado por cem.

A média anual de consultas médicas por habitante nas especialidades médicas básicas é calculada por meio da divisão do número de consultas médicas nas especialidades básicas realizados em cada município no ano de 2007 pelo total da população no mesmo ano e período. A média mensal de visitas domiciliares por família é a quantidade de visitas realizadas pelos membros de equipes de saúde da família divididas pelo número de famílias no município vezes o número de meses (12 meses). Esse número de famílias é calculado dividindo-se a população residente no município pelo número médio de pessoas por família (3,3 pessoas). 
A cobertura de primeira consulta odontológica é calculada pela divisão do número total de primeiras consultas odontológicas programáticas realizadas no município no ano de 2007 , pela população total do mesmo local e período e, posteriormente multiplicados por cem.

A cobertura da ação coletiva de escovação dental supervisionada é calculada através da divisão entre a média de pessoas participantes na ação coletiva denominada "escovação dental supervisionada" realizada em determinado local no ano de 2007 pela população total estimada no mesmo período e posteriormente, multiplicada por cem. Esse indicador expressa o percentual de cobertura correspondente à média de pessoas que tiveram acesso à escovação dental com orientação/supervisão de um profissional de saúde, considerando o mês ou meses em que se realizou a atividade, em determinado local e ano. Este indicador também permite estimar a proporção de pessoas que teve acesso ao flúor tópico proveniente do dentifrício através do serviço público de saúde.

Os procedimentos odontológicos básicos são entendidos como a divisão do número de procedimentos odontológicos básicos individuais realizados em cada município no ano selecionado pelo total da população no mesmo ano e período. Por último, a proporção de procedimentos odontológicos especializados em relação às ações odontológicas individuais consiste na proporção de procedimentos odontológicos especializados em relação às demais ações odontológicas individuais realizadas no âmbito do Sistema Único de Saúde.

A análise descritiva foi realizada para todas as variáveis em questão. A análise de segmentação, denominada Cluster Hierárquico, foi realizada para segmentação dos municípios a partir dos indicadores socioeconômicos - Índice de Gini, IDH-M, IMRS e taxa de alfabetização. Para este estudo o método de agrupamento foi Between-linkage group e a medida de dissimilaridade entre os municípios foi a Distância Euclidiana Quadrática.

A análise de segmentação foi executada em dois momentos. No primeiro, todas as variáveis correspondentes aos indicadores municipais foram inseridas no modelo ao mesmo tempo para os 34 municípios da amostra. Obtiveram-se dois clusters, um grupo formado apenas pela capital Belo Horizonte e outro grupo com todos os 33 municípios da RMBH. Entretanto, nesse método de análise, não é possível fazer inferências para um agrupamento (cluster) com apenas um elemento ${ }^{16}$. Por essa razão, para o presente estudo, a capital foi excluída da amostra e uma nova análise foi realizada com todos os indicadores em uma amostra de 33 municípios.

A construção do banco de dados foi realizada no programa estatístico Statistical Package for the Social Science for Windows - SPSS versão 17 para análise dos mesmos. A comparação dos indicadores de saúde da Atenção Básica entre os clusters foi feita através do teste $t$-Student, com nível de significância estabelecido em $\mathrm{p}<0,05$.

\section{RESULTADOS}

Formaram-se dois clusters distintos, sendo que $23(70 \%)$ municípios foram classificados como de piores indicadores socioeconômicos (cluster 1) e 10 (30\%) como de melhores indicadores (cluster 2).

A Tabela 1 mostra as medidas de resumo para os indicadores socioeconômicos e de saúde da Atenção Básica. RMBH, 2009. As maiores diferenças entre os valores mínimo e máximo foram para a cobertura da Saúde da Família $(107,58)$, média de visita domiciliar $(98,99)$ e procedimentos especializados $(98,62)$.

Os indicadores socioeconômicos dos municípios agrupados nos dois clusters estão apresentados na Tabela 2.

A Tabela 3 mostra que não haver diferença das médias de consultas médicas entre os clusters $(2,19 \pm 0,99$ para cluster 1 e $2,01 \pm 0,94$ para o cluster 2). Por outro lado, a média do indicador de visita domiciliar do cluster $1(6,08 \pm 24,57)$ foi cerca de sete vezes maior que a do cluster $2(0,80 \pm 0,64)(\mathrm{p}=$ $0,049)$. Também, foi observado maior cobertura pela Estratégia de Saúde da Família no cluster 1 quando comparado com o cluster $2(p=0,049)$.

Em relação aos indicadores de Saúde Bucal, não houve diferenças estatisticamente significantes entre os clusters. $\mathrm{O}$ grupo com melhores indicadores socioeconômicos obteve médias superiores ao outro grupo, com exceção à cobertura da primeira consulta odontológica. A maior discrepância entre os clusters 1 e 2 foi na cobertura de escovação supervisionada 11,52 e 15,37, respectivamente (Tabela 4). 
Tabela 1 - Medidas de resumo para os indicadores socioeconômicos e de saúde da Atenção Básica. RMBH, 2009

\begin{tabular}{llllllllllll}
\hline & IDH-M & $\begin{array}{l}\text { TAXA } \\
\text { ALFA- } \\
\text { BETIZ. }\end{array}$ & $\begin{array}{l}\text { ÍNDICE } \\
\text { GINI }\end{array}$ & IMRS & $\begin{array}{l}\text { COB. } \\
\text { ESF }\end{array}$ & $\begin{array}{l}\text { CONS. } \\
\text { MÉDICA }\end{array}$ & $\begin{array}{l}\text { VISITA } \\
\text { DOMI- } \\
\text { CILIAR }\end{array}$ & $\begin{array}{l}\text { COB. } \\
\text { ODON- } \\
\text { TO }\end{array}$ & $\begin{array}{l}\text { COB. } \\
\text { ESCO- } \\
\text { VAÇÃO }\end{array}$ & $\begin{array}{l}\text { PROC. } \\
\text { BÁSICO }\end{array}$ & $\begin{array}{l}\text { PROC. } \\
\text { ESPEC. }\end{array}$ \\
\hline Mínimo & 0,70 & 82,52 & 0,44 & 0,48 & 1,19 & 0,66 & 0,01 & 0,88 & 0,01 & 0,10 & 0,38 \\
Percentil 25 & 0,74 & 87,97 & 0,49 & 0,58 & 56,93 & 1,36 & 0,36 & 4,52 & 1,91 & 0,32 & 1,21 \\
Média & 0,75 & 89,40 & 0,52 & 0,62 & 73,23 & 2,07 & 3,71 & 10,54 & 12,71 & 0,80 & 13,58 \\
(DP) & $( \pm 0,03)$ & $( \pm 3,04)$ & $( \pm 0,05)$ & $( \pm 0,06)$ & $( \pm 29,80)$ & $( \pm 0,90)$ & $( \pm 17,11)$ & $( \pm 8,11)$ & $( \pm 13,81)$ & $( \pm 0,67)$ & $( \pm 21,83)$ \\
Mediana & 0,75 & 90,43 & 0,51 & 0,63 & 85,46 & 1,83 & 0,81 & 9,75 & 10,29 & 0,65 & 5,22 \\
Percentil 75 & 0,77 & 91,55 & 0,56 & 0,66 & 99,00 & 2,91 & 1,06 & 14,07 & 16,71 & 1,14 & 15,36 \\
Máximo & 0,82 & 94,73 & 0,64 & 0,73 & 108,77 & 3,91 & 99,00 & 32,29 & 58,75 & 3,35 & 99,00 \\
\hline
\end{tabular}

IMRS: Índice Mineiro de Responsabilidade Social; IDH-M: Índice de Desenvolvimento Humano Municipal; Cob. ESF: cobertura Estratégia de Saúde da Família; Cons. Médica: média de consulta médica; Visita Domiciliar: média de visita domiciliar; Cob Odonto: cobertura da $1^{\text {a }}$ consulta odontológica programática; Cob Escovação: cobertura da ação coletiva de escovação dental supervisionada; Proc. Básico: média de procedimentos odontológicos básicos individuais; Proc. Espec.: proporção de procedimentos odontológicos especializados em relação às ações odontológicas individuais.

Tabela 2 - Média e desvio padrão das variáveis utilizadas no agrupamento dos clusters em relação aos indicadores socioeconômicos. RMBH, 2009

\begin{tabular}{lllll}
\hline & IDH-M & IMRS & ÍNDICE GINI & TAXA DE ALFABETIZAÇÃO \\
\hline Cluster $\mathbf{1}(\mathrm{N}=23)$ & $0,74( \pm 0,02)$ & $0,60( \pm 0,03)$ & $0,51( \pm 0,04)$ & $88,29( \pm 2,86)$ \\
Cluster 2 $(\mathrm{N}=10)$ & $0,79( \pm 0,02)$ & $0,69( \pm 0,05)$ & $0,55( \pm 0,05)$ & $91,97( \pm 1,55)$ \\
\hline
\end{tabular}

IMRS: Índice Mineiro de Responsabilidade Social

IDH-M: Índice de Desenvolvimento Humano Municipal

Tabela 3 - Média e desvio padrão dos indicadores Gerais da Atenção Básica analisados segundo a classificação dos indicadores socioeconômicos. RMBH, 2009

\begin{tabular}{llll}
\hline & COB ESF (percentual médio) & CONS. MÉDICA & VISITA DOMICILIAR \\
\hline Cluster $\mathbf{1}(\mathrm{N}=23)$ & $77,62( \pm 32,45)^{*}$ & $2,19( \pm 0,99)$ & $6,08( \pm 24,57)^{*}$ \\
Cluster $\mathbf{2}(\mathrm{N}=10)$ & $70,42( \pm 27,86)^{*}$ & $2,01( \pm 0,94)$ & $0,80( \pm 0,64)^{*}$ \\
\hline
\end{tabular}

* Diferença entre os clusters estatisticamente significante $(\mathrm{p}=0,049)$.

COB ESF: cobertura Estratégia de Saúde da Família

CONS. MÉDICA: média de consulta médica

VISITA DOMICILIAR: média de visita domiciliar

Tabela 4 - Média e desvio padrão dos indicadores Gerais da Atenção Básica analisados segundo a classificação dos indicadores socioeconômicos. RMBH, 2009

\begin{tabular}{lllll}
\hline & COB ODONTO* & COB ESCOVAÇÃO* & PROC. BÁSICO* & PROC. ESPEC.* \\
\hline Cluster 1 $(\mathrm{N}=23)$ & $9,27( \pm 7,94)$ & $11,52( \pm 12,07)$ & $0,66( \pm 0,40)$ & $11,39( \pm 24,09)$ \\
Cluster 2 $(\mathrm{N}=10)$ & $9,21( \pm 7,94)$ & $15,37( \pm 19,14)$ & $0,72( \pm 0,46)$ & $11,96( \pm 10,65)$ \\
\hline
\end{tabular}

* Não houve diferença estatisticamente significante entre os clusters

COB ODONTO: cobertura da $1^{\text {a }}$ consulta odontológica programática

COB ESCOVAÇÃ̃O: cobertura da ação coletiva de escovação dental supervisionada

PROC. BÁSICO: média de procedimentos odontológicos básicos individuais

PROC. ESPEC.: proporção de procedimentos odontológicos especializados em relação às ações odontológicas individuais.

\section{DISCUSSÃO}

A primeira limitação deste estudo diz respeito ao tamanho da amostra $(\mathrm{N}=33)$ que, apesar de possibilitar a utilização do método hierárquico de segmentação, não permite outras comparações entre os clusters além de análises bivariadas.

A análise de cluster pressupõe a formação de um conjunto de objetos no qual cada elemento está mais próximo e é mais similar a elementos dentro do cluster do que qualquer outro fora do grupo. O tipo
Hierárquico produz automaticamente agrupamentos começando com clusters aninhados e unitários e repetidamente aglutinando clusters próximos dois a dois até chegar ao número de clusters pertinente ${ }^{16}$.

Durante a análise, o município de Belo Horizonte permaneceu isolado até o final do processo. Sendo assim, a exclusão da capital da análise possibilitou que um melhor agrupamento entre os outros municípios fosse possível. Apesar de o Método Hierárquico ser o mais adequado para 
amostras pequenas, possui uma limitação importante durante o agrupamento dos pares de casos. Um caso só será agrupado ao cluster se ele estiver ligado à, pelo menos, mais um caso ${ }^{16}$.

Os problemas relacionados à utilização de dados secundários são estabelecidos na literatura, tais como fragilidades dos registros e a dificuldade do controle de qualidade dessas informações nas esferas Estadual e Federal. Ainda assim, são estudos importantes que podem auxiliar no planejamento das ações dos serviços de saúde.

Neste estudo, os indicadores sociais foram escolhidos para agrupar os municípios pela análise de segmentação por contemplarem os aspectos socioeconômicos de maneira combinada. Porém, outras variáveis contextuais como os aspectos demográficos, aspectos relacionados à infraestrutura física e aos recursos humanos dos serviços de saúde e o investimento público em saúde não foram avaliadas. Isso porque essas informações não estão disponíveis para todos os municípios da RMBH. Além disso, os indicadores selecionados correspondem aos resultados do censo de 2000; uma vez que houve apenas uma divulgação parcial e agregada dos dados do censo de 2010.

Essa amostra pode ser considerada relativamente homogênea, pois os clusters apresentaram alta heterogeneidade externa, mas a homogeneidade interna não pôde ser medida. Além disso, tratam-se de municípios que pertencem à mesma Região Metropolitana com indicadores sociais bem semelhantes ${ }^{17}$, especialmente o IDH.

Por outro lado, a análise de segmentação foi uma ferramenta muito útil para esse tipo de amostra, pois pequenas particularidades de cada município apareceram durante o processo de agrupamento em diferentes clusters. A análise de segmentação é um procedimento multivariado para detectar clusters homogêneos em um conjunto de dados ${ }^{16}$. Entretanto, como não há conhecimento a priori da alocação dos casos (municípios) nos clusters, a interpretação dos resultados deve ser feita com cautela para serem usados como exploração de possíveis relações entre as variáveis em questão (indicadores socioeconômicos).

O cluster 2 reuniu os municípios com os melhores indicadores socioeconômicos, ou seja, maiores índices de desenvolvimento humano, IMRS e taxa de alfabetização. Entretanto, uma ressalva deve ser feita em relação ao índice de Gini, o qual foi maior no cluster 2, indicando que há uma maior desigualdade na distribuição de indivíduos segundo a renda domiciliar per capita nestes municípios. A análise de segmentação hierárquica não realiza testes de associação entre os clusters, mas por meio de suas matrizes é possível identificar as diferenças entre os clusters formados. As variáveis (IDH, IMRS, Índice
Gini e Taxa de Alfabetização) inseridas no modelo são utilizadas apenas para identificar semelhanças e diferenças entre os municípios, a fim de separá-los em grupos distintos, denominados clusters. Após a formação dos clusters, outros testes podem ser utilizados para mostrar diferenças entre os clusters formados e variáveis de interesse. O presente estudo utilizou o teste $t$-Student para medir as diferenças entre os clusters em relação aos indicadores da Atenção Básica.

A análise de segmentação agrupou os municípios em dois clusters a partir das diferenças dos indicadores socioeconômicos entre os mesmos. Entretanto, quando os clusters foram comparados em relação aos indicadores de saúde da Atenção Básica, constataram-se diferenças estatisticamente significantes apenas para a cobertura pela Estratégia de Saúde da Família e para a média de visita domiciliar. Os municípios com piores indicadores socioeconômicos (cluster 1) apresentaram maior cobertura pela ESF e maior média mensal de visitas domiciliares por família. Apesar dos estudos com análise de segmentação ser incipiente na área da saúde, esse resultado é semelhante ao observado no Brasil quando se estratificou os municípios por IDH-M, sendo que quanto mais baixo o IDH-M maior o nível de cobertura pela $\mathrm{ESF}^{1}$.

O impacto da ESF nos indicadores de saúde permanece controverso $^{19}$, ainda assim, é válido ressaltar que a estratégia ainda está em construção e necessita de qualificação para concretizar a mudança do modelo assistencial e obter resultados mais expressivos $^{20}$.

A cobertura de Equipe de Saúde da Família e o número de visitas domiciliares foram maiores nos municípios com piores indicadores socioeconômicos, sendo esta uma política do próprio Ministério da Saúde $^{1}$, que visa priorizar o aumento das equipes nos municípios com menores recursos financeiros. Faz parte da estratégia do Ministério da Saúde incentivar maior cobertura e ações de promoção e recuperação da saúde nos municípios com piores indicadores socioeconômicos, através de políticas de saúde efetivas e possibilitadoras de mudanças ${ }^{5}$.

O Brasil é um país com inúmeras realidades sociais e demográficas, além de processos diferenciais de organização da assistência à saúde, portanto é plausível haver diferenças na implantação da Estratégia de Saúde da Família evidenciada nas diferenças entre seus indicadores de saúde ${ }^{20}$. Para os indicadores da saúde bucal essas diferenças não foram observadas.

Torna-se importante ressaltar que outros fatores como os demográficos, por exemplo, devem ser considerados em estudos que avaliam indicadores sociais e de renda por meio de clusters baseados na 
média populacional, uma vez que tais fatores podem alterar a homogeneidade interna desses clusters.

Apesar das limitações anteriormente discutidas, os resultados do presente estudo sugerem haver maior cobertura pelo programa de Estratégia da Saúde da Família e, também, maior número de visitas domiciliares em municípios com piores indicadores socioeconômicos.

Sugere-se a realização de um estudo ecológico com todos os municípios mineiros ou ainda um estudo com cálculo amostral aleatório proporcional dos municípios para consolidar os resultados encontrados no presente estudo.

\section{ABSTRACT}

Aim: To investigate the association between socioeconomic indicators and Primary Health Care indicators of the Brazilian Health Care System in the greater Belo Horizonte Metropolitan Area, in the state of Minas Gerais, Brazil. Materials and Methods: Ecological study based on secondary data from the Belo Horizonte Metropolitan Area ( $\mathrm{N}=33$ municipalities). The independent variables included the overall health and oral health indicators from Primary Health Care. The exploratory variables included the literacy rate, Human Development Index, Gini Index, and Social Responsibility Index of the state of Minas Gerais. The Hierarchical Cluster analysis was conducted to subdivide the municipalities according to socioeconomic indicators, using the Between-linkage group method and the Squared Euclidean distance measure. The Primary Health Care indicators were compared among clusters by means of Student's t tests with a significance level of 5\%. Results: The municipalities were grouped in two clusters: 1) worst $(\mathrm{N}=23)$ and 2) best $(\mathrm{N}=10)$ socioeconomic indicators. Cluster 1, as compared to cluster 2, presented a higher coverage of the Family Healthcare Strategy and a higher average of doctors' house calls (76.6 \pm $32.5 ; 6.1 \pm 24.6$, respectively) $(70.4 \pm 27.9 ; 0.8 \pm 0.6$, respectively) $(\mathrm{p}<0.05)$. Concerning the oral health indicators, no statistically significant difference could be observed among the clusters. Conclusion: The results suggest an association among the municipalities of the greater Belo Horizonte Metropolitan Area which present worse socioeconomic indicators and a higher coverage of the Family Healthcare and average of doctors' house calls.

Descriptors: Primary health care. Health status indicators. Social indicators.

\section{AGRADECIMENTOS}

Os autores agradecem à FAPEMIG pelo apoio financeiro (Processo APQ-01734-09). Agradecem, ainda, a Giovane Viana Campos pela participação na coleta de dados.

\section{REFERÊNCIAS}

1. Brasil. Ministério da Saúde. Secretaria de Atenção à Saúde. Departamento de Atenção Básica. Saúde da família no Brasil: uma análise de indicadores selecionados: 1998-2005/2006 [Internet]. Brasília: Ministérioda Saúde, 2008. [acesso em 2010 nov24]. Disponível em: http://189.28.128.100/dab/docs/ publicacoes/geral/saude_familia_no_brasil_uma analise_indicadores_selecionados_1998_2006. pdf

2. Norma Operacional Básica do SUS/NOB-SUS 01/96. Gestão plena com responsabilidade pela saúde do cidadão [Internet]. Brasília: Ministério da Saúde, 1997. Publicada no D.O.U.de 6/11/1996. [acesso em 2010 nov 24]. Disponível em: http:// www.portalsocial.ufsc.br/legislacao/saude/nob96. pdf

3. Brasil. Ministério da Saúde. Portaria no 493 de 13 de março de 2006. Aprova a Relação de Indicadores da Atenção Básica - 2006, cujos indicadores deverão ser pactuados entre municípios, estados e o Ministério da Saúde [Internet]. [acesso em 2010 dez 12]. Disponível em: http://tabnet.datasus.gov. br/cgi/siab/pacto2006/portaria_493.pdf

4. Buss PM, Pellegrini Filho A. A saúde e seus determinantes sociais. Physis. 2007; 17:77-93.

5. Fernandes LS, Peres MA. Associação entre atenção básica em saúde bucal e indicadores socioeconômicos municipais. Rev Saúde Pública. 2005; 39:930-6.

6. Junqueira SR, Araújo ME, Antunes JLF, Narvai PC. Indicadores socioeconômicos e recursos odontológicos em municípios do estado de São Paulo, Brasil, no final do século XX. Epidemiol Serv Saúde. 2006; 15:41-53.

7. Fischer TK, Peres KG, Kupek E, Peres MA. Indicadores de atenção básica em saúde bucal: associação com as condições socioeconômicas, provisão de serviços, fluoretação de águas e a estratégia de saúde da família no Sul do Brasil. Rev Bras Epidemiol. 2010; 13:126-38.

8. Aquino R, Barreto ML. Programa Saúde da Família: acerca da adequação do uso do seu indicador de cobertura. Cad Saúde Pública. 2008; 24:905-14.

9. Machado CC. Indicadores sócio-demográficos: Belo Horizonte e região metropolitana. In: Prefeitura Municipal de Belo Horizonte. Anuário estatístico 2000 [Internet]. [acesso em $2010 \mathrm{dez}$ 12]. Disponível em: http://portalpbh.pbh.gov.br/ $\mathrm{pbh} / \mathrm{ecp} / \mathrm{comunidade} \cdot \mathrm{do}$ ?evento=portlet\&pIdPlc= 
ecpTaxonomiaMenuPortal\&app=estatisticas\&tax $=11807 \&$ lang $=$ pt_BR\&pg $=5922 \& \operatorname{taxp}=0 \&$

10. Luz ZMP, Pimenta DN, Cabral LLV, Fiúza VOP, Rabello A. A urbanização das leishmanioses e a baixa resolutividade diagnóstica em municípios da região metropolitana de Belo Horizonte. Rev Soc Bras Med Trop. 2001; 34:249-54.

11. Velásquez-Meléndez G, Pimenta AM, Kac G. Epidemiologia do sobrepeso e da obesidade e seus fatores determinantes em Belo Horizonte (MG), Brasil: estudo transversal de base populacional. Rev Panam Salud Publica. 2004; 16:308-14.

12. Lima-Costa MF. Estilos de vida e uso de serviços preventivos de saúde entre adultos filiados ou não a plano privado de saúde (inquérito de saúde de Belo Horizonte). Ciênc Saúde Coletiva. 2004; 9:857-64.

13. Lima-Costa MF. Fatores associados à vacinação contra gripe em idosos na região metropolitana de Belo Horizonte. Rev Saúde Pública. 2008; 42:1007.

14.Laschefski K, Costa HSM. Segregação social como externalização de conflitos ambientais: a elitização do meio ambiente na APA-Sul, região metropolitana de Belo Horizonte. Ambient Soc. 2008; 11:307-22.

Recebido em 25/05/2011 - Aceito em 10/10/2011

Autor correspondente:

Ana Cristina Viana Campos

Rua dos Jés, 151/302 - Santa Mônica

CEP: 31.530-160 - Belo Horizonte - MG - Brasil

E-mail: campos.acv@gmail.com
15. Luz TCB, Loyola Filho AI, Lima-Costa MF. Estudo de base populacional da subutilização de medicamentos por motivos financeiros entre idosos na região metropolitana de Belo Horizonte, Minas Gerais, Brasil. Cad Saúde Pública. 2009; $25: 1578-86$.

16. Hair JF, Black WC, Babin JB, Anderson RE, Tatham RL. Análise multivariada de dados. $6^{\mathrm{a}}$ ed. Porto Alegre: Artmed; 2009.

17. Gôuvea RG. Diretrizes para a gestão metropolitana no Brasil. Eure. 2009; 104:47-76.

18. Conill EM. Ensaio histórico-conceitual sobre a atenção primária à saúde: desafios para a organização de serviços básicos e da Estratégia Saúde da Família em centros urbanos no Brasil. Cad Saúde Pública. 2008; 24 Suppl 1:S7-27.

19. Gomes KO, Cotta RMM, Euclydes MP, Targueta CL, Priore SE, Franceschini SCC. Avaliação do impacto do Programa Saúde da Família no perfil epidemiológico da população rural de Airões, município de Paula Cândido (MG), 1992-2003. Ciênc Saúde Coletiva. 2009; 14 Suppl 1:1473-82.

20. Campos FE, Aguiar RAT, Oliveira VB. O desafio da expansão do programa de Saúde da Família nas grandes capitais brasileiras. Physis. 2002; 12:4758. 